\title{
MS40-03 | Nano-Scale Strain Mapping in Complete Nanowire Based Electronic Devices by Bragg Coherent X-Ray Diffraction
}

Dzhigaev, Dmitry (Lund University, Lund, SWE)

The progress achieved in a growth of heterostructured nanowires led to design of nano-electronic devices with record-breaking parameters in transistors, quantum computations, solar cells and light emitting technologies. The strain plays significant role in the performance of these nano-electronic devices. In multi-segment nanowires the strain induced at the interfaces significantly change a charge carrier mobility, thus alternating the speed and power consumption during operation. The only way to image the strain in real functional device with many functional layers and over extended field of view is to employ scanning techniques with the use of nano-focused X-ray beams.

We made a deeper look at nanoscale features within single heterostructured nanowires, which are embedded in realistic devices. These are InAs/GaSb segments of TFETs, lateral nanowires of InGaAs MOSFETs and $\mathrm{GaAs} / \mathrm{InGaAs} / \mathrm{InAs}$ nanowires for quantum computation applications. Exploiting extraordinary brilliance of $4^{\text {th }}$ generation synchrotron MAX IV (Lund, Sweden) and dedicated nano-focusing end-station NanoMax we could obtain the information which was not accessible before at resolution of $60 \mathrm{~nm}$ and below. Development of Bragg diffraction mapping and Bragg ptychography techniques in recent years opened exciting possibilities for 3D imaging of strain and tilt in various structures. These techniques were employed during our studies. The experimental challenges will be discussed in more detail. Interpreting of the results plays a significant role in obtaining quantitative information, which includes developing of proper models for strain engineering in nanoelectronics. 